RESEARCH ARTICLE

\title{
Biased epitope selection by recombinant vaccinia-virus (rVV)-infected mature or immature dendritic cells
}

\author{
D Nagorsen ${ }^{1,2}$, M Panelli $^{1}$, ME Dudley ${ }^{3}$, SE Finkelstein ${ }^{3}$, SA Rosenberg ${ }^{3}$ and FM Marincola ${ }^{1}$ \\ ${ }^{1}$ Immunogenetics Section, Department of Transfusion Medicine, Clinical Center, National Institutes of Health, Bethesda, MD, USA; \\ ${ }^{2}$ Home Institution, Med.Klinik III, UKBF, FU Berlin, Germany; and ${ }^{3}$ Surgery Branch, National Cancer Institute, National Institutes of \\ Health, Bethesda, MD, USA
}

Recombinant expression vectors represent a powerful way to
deliver whole antigens (Ags) for immunization. Sustained Ag
expression in vector-infected dendritic cells (DC) combines
Ag-specific stimulation with powerful costimulation and,
simultaneously, through 'self-selection' of ad hoc epitopes
broadens the scope of immunization beyond restrictions
posed by individual patients' human leukocyte antigen (HLA)
phenotype. In this study, therefore, we evaluated the efficiency
of a recombinant vaccinia virus encoding the gp100/PMel17
melanoma Ag (rVV-gp100) to infect immature (iDC) or mature
dendritic cells (mDC) derived from circulating mononuclear
cells and the effect of infection on their status of maturation. In
addition, we tested the ability of rVV-gp100-infected iDC and
$m D C$ to present the HLA-A*0201-associated gp100:209-217
epitope (g209). Irrespective of status of maturation, rVV-
gp100 infection induced gp100 expression while only partially
reversing the expression of some maturation markers.

However, endogenous presentation of the wild-type g209 epitope was inefficient. The low efficiency was epitope-specific since infection of DC with rVV encoding a gp100 construct containing the modified gp100:209-217 (210M) (g209-2M) epitope characterized by high binding affinity for HLA-A*0201 restored efficient $\mathrm{Ag}$ presentation. Presentation of an $H L A-$ class II-associated epitope and cytokine release by $D C$ was not altered by rVV infection. Thus, Ag expression driven by rVV may be an efficient strategy for whole Ag delivery. However, since the effectiveness of $\mathrm{Ag}$ processing and presentation is subject to stringent HLA/epitope pairing, and for other yet undefined rules, the assumption that whole $\mathrm{Ag}$ delivery may circumvent HLA restriction is incorrect and recombinant expression vectors encoding well-characterized polyepitopic constructs may prove more effective.

Gene Therapy (2003) 10, 1754-1765. doi:10.1038/ sj.gt.3302066

Keywords: antigen; CTL; dendritic cells; epitopes; $M H C$; vaccinia virus

\section{Introduction}

Professional antigen presentation by dendritic cells (DC) may play a central role in enhancing the effectiveness of antigen-(Ag) specific immunization toward viral and tumor targets. ${ }^{1,2}$ In natural conditions, DC most likely present exogenous Ag up-take from dead cells. ${ }^{3,4}$ However, for therapeutic purposes, endogenous presentation of Ag encoded by viral vectors infecting DC has been proposed in murine ${ }^{5}$ and human ${ }^{6-9}$ models. This strategy of Ag delivery can induce human leukocyte antigen (HLA) class I-and class II-associated immune responses and may represent a potent immunization tool inducing prolonged Ag-specific stimulation within cells capable of powerful costimulation. In addition, wholeprotein expression by vector-infected DC may enhance the immunogenicity of subdominant epitopes and, consequently, broaden the scope of immunization beyond restrictions posed by the HLA polymorphism.

Immunization through endogenous induction of whole Ag is based on the assumption that the epitope repertoire of a given $\mathrm{Ag}$ can be adjusted to various HLA

Correspondence: Dr FM Marincola, Department of Transfusion Medicine, Clinical Center, National Institute of Health Building 10, Room 1C711, 10 Center Drive, Bethesda, MD 20892-1502, USA

Received 6 September 2002; accepted 7 March 2003 phenotypes by a process of 'self-selection' whereby peptides are naturally coupled according to binding affinity to the HLA alleles expressed by a given individual. Although theoretically undisputable, in practice the truth of this assumption is dependent on the efficiency with which individual molecules are processed and presented in association with HLA alleles. Since most human tumor Ag are nonmutated selfproteins, $\mathrm{T}$ cells recognizing peptides with high affinity for HLA-class I alleles may have undergone negative selection during embryogenesis and only moderate- to low-affinity peptides are potential cytotoxic T-cell epitopes. ${ }^{10}$ This appears to be the case for the two melanoma differentiation Ag MART-1/MelanA (MART-1) and gp100/PMel17 (gp100), whose commonly recognized epitopes in the context of HLA-A*0201 allele are characterized by low-to-medium binding affinity for this allele. ${ }^{11,12}$ While both Ag are natural targets of effector $\mathrm{T}$ cells, both appear suboptimal for the T-cell activation required for priming an immune response in the context of immunization. Thus, several groups have identified analog heteroclitic peptides characterized by higher binding affinity for HLA-A*0201 and stronger immunogenicity in vitro ${ }^{13-16}$ and upon in vivo administration. ${ }^{17,18}$

In this context, the ability of DC to present relevant epitopes is a prerequisite for the specificity of their immunogenic function as other facilitator mechanisms - such as 
expression of costimulatory molecules or release of soluble factors with immune stimulatory or chemoattractant properties - cannot substitute for Ag-specific signaling. Thus, in this study, we tested the kinetics of epitope/TCR interactions as an essential component of the equation leading to efficient Ag presentation by DC. In particular, we analyzed the efficiency of DC to engage HLA-class I-restricted TCR by presenting naturally occurring epitopes. Previous work showed that the gp100:209-217 (g209) epitope represents a natural target for tumor-specific effector memory tumor infiltrating lymphocytes (TIL) characterized by an effector-memory phenotype. However, the same epitope does not efficiently prime T-cell responses in vitro and in vivo compared with the modified gp100:209-217 (210M) (g209-2M) epitope characterized by higher binding affinity for HLA-A*0201. ${ }^{17,18}$ Owing to the importance of intensity and persistence of Ag-specific stimulation for induction of primary immune responses, ${ }^{19,20}$ we tested the ability of DC to process and present endogenous gp100. In particular, we compared the efficiency of endogenous Ag-specific presentation by DC to (1) that of tumor cells to represent the targets for activated effector memory cells and (2) that of DC loaded with saturating amounts of the g209-2M heteroclitic epitope to represent the stimulus most likely associated with successful immunization. ${ }^{17}$

Although Ag-specific stimulation is an essential component of the adaptive immune response, the efficiency of DC as professional Ag-presenting cells is determined by other factors that could not be disregarded in this study. Maturation of DC appears to be a prerequisite for efficient activation of viral particlespecific CD8+ T-cells ${ }^{21}$ since mature DC (mDC) can induce functionally active IFN- $\gamma$ producing $\mathrm{CD}^{+} \mathrm{T}$-cells while immature DC (iDC) induce more regulatory, IL10-producing $\mathrm{CD}^{+}$and $\mathrm{CD}^{+}{ }^{+} \mathrm{T}_{\text {-cells. }}{ }^{22}$ Indeed, there is preliminary evidence that $\mathrm{mDC}$ induce stronger $\mathrm{T}$-cell responses than iDC in cancer patients. ${ }^{23}$ However, some viruses commonly used as vectors for the delivery of $\mathrm{Ag}$ such as recombinant vaccinia -viruses (rVV) may inhibit the maturation of DC as a mechanism of immune evasion. ${ }^{24}$ Thus, although rVV can induce strong immune responses, it remains unclear how these develop in the infected host. Pox viruses employ many mechanisms to evade the immune system, such as producing proteins that oppose apoptosis, synthesize steroids, capture chemokines, counteract complement, obstruct interferon function or intercept interleukins. ${ }^{25}$ Thus, for practical purposes, it remains important to understand how infection with rVV of mDC or iDC affects the Agspecific and nonspecific functions of these important AG-presenting cells.

The effect of rVV infection on maturation-related functions of DC was first assessed. In addition, the ability of presenting HLA-class II-associated epitopes was confirmed. Since none of them appeared to be strongly affected in this experimental model, we turned our attention to the primary goal of the study. Thus, we compared the efficiency of an rVV encoding gp100 (rVVgp100) to infect iDC and $\mathrm{mDC}$ derived from circulating mononuclear cells. We also compared the ability of iDC and $\mathrm{mDC}$ cells to present the wild-type rVV-induced gp100 epitope g209 versus the exogenously provided epitope g209-2M to CD8+ g209-specific CTL clones.
Finally, we tested whether epitope processing and presentation were altered by rVV infection or were rather dependent on the biochemical characteristics of the individual epitope. This was explored by infecting iDC and mDC with an rVV encoding a modified gp100 construct containing the g209-2M epitope. The results suggest that Ag-specific, HLA-class I-associated presentation by DC is highly dependent on the biochemical affinity of individual epitopes for their respective HLA allele and, therefore, several known natural epitopes may not be efficiently expressed even in the context of ideal costimulation.

\section{Results}

Maturation of DC and effect of rVV-gp100 infection on viability: maturation, and gp100 expression in iDC and $m D C$

As expected, after 10 independent in vitro maturations, phenotype and functional aspects of $\mathrm{mDC}$ and iDC demonstrated that HLA-DR and CD86 were comparably highly expressed, while mDC expressed consistently and significantly more CD80 mean fluorescence intensity (MFI $=22.7 \pm 1.4$ versus $13.4 \pm 1.7, P_{2}<0.001$, paired Student's $t$-test) and CD83 (MFI $=15.0 \pm 1.9$ versus $7.1 \pm 0.6$, $P_{2}<0.01$, paired Student's $t$-test) and had lost endocytotic function while becoming potent stimulators of IFN- $\gamma$ secretion in response to allostimulation. Thus, treatment with CD40L and LPS was considered a satisfactory model for the preparation of two functionally distinct subsets of DC.

In paired experiments, $\mathrm{mDC}$ and $\mathrm{iDC}$ were then exposed to rVV-gp100 at 1:10 multiplicity of infection (MOI). After overnight incubation, the DC were tested for viability according to the trypan blue exclusion method. No significant differences were noted in viability in infected versus noninfected $\mathrm{iDC}$ and $\mathrm{mDC}$ (iDC $84.1 \pm 1.9$ versus $\mathrm{iDC}+\mathrm{rVV} 76.3 \pm 2.7$ and $\mathrm{mDC}$ $90.0 \pm 2.5$ versus $\mathrm{mDC}+\mathrm{rVV} 83.3 \pm 2.3$, average percent of viable cells in six consecutive experiments). The cells were then fixed and stained with the anti-gp100 mAb HMB-45 to indirectly test for $\mathrm{rVV}$ infection through the expression of the relevant gene. In nine consecutive experiments, no differences were noted between the two cell populations in the ability of rVV to infect and induce expression of gp100 (Figure $1 \mathrm{a}$ and $\mathrm{b}$ ). No significant differences were noted in the expression of gp100 in the two DC populations, whether percentage of gp100 expressing cells or intensity of gp100 expression in infected cells was analyzed. In average $36.4 \pm 3.9 \%$ of iDC and $34.0 \pm 3.2 \%$ of $\mathrm{mDC}$ demonstrated expression of gp100 (Figure 1c), a figure similar to our previous experience using this infection protocol. ${ }^{8}$ Cells demonstrating evidence of gp100 expression were separately gated and assessed for intensity of expression of the melanoma Ag. No significant differences were noted in intensity of gp100 expression between iDC and mDC (Figure 1d), nor with the constitutive level of expression of gp100 by the SK23 melanoma cell line (data not shown). Thus, the status of maturation of DC did not appear to influence the ability of rVV to infect these cells and induce the expression of virally driven proteins. 


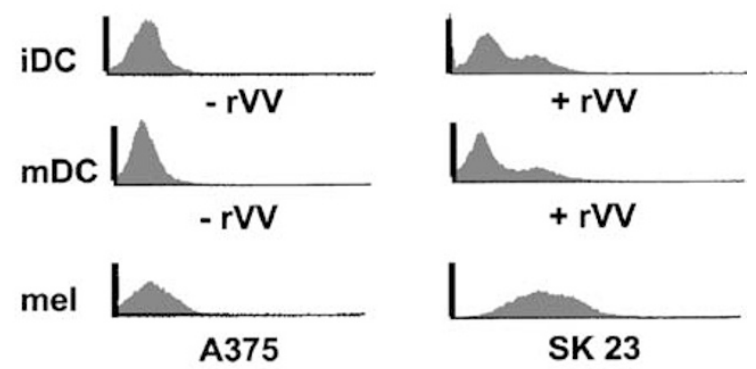

b

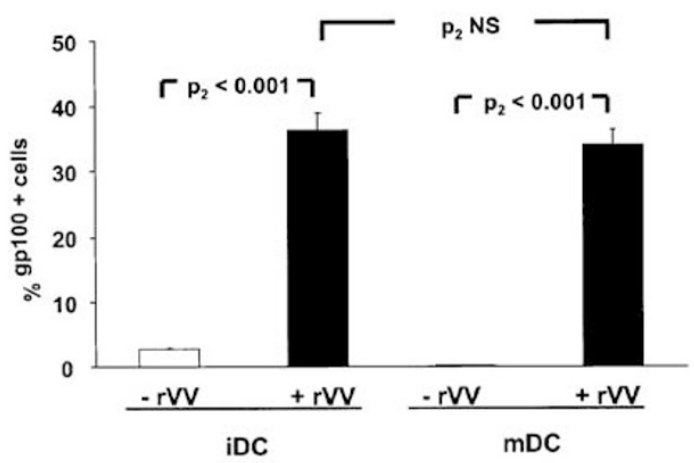

C

\begin{tabular}{lcccc}
\hline experiment & iDC $-r V V$ & iDC $+r V V$ & $m D C-r V V$ & $m D C+r V V$ \\
Exp 1 & 2.1 & 40.9 & 0.0 & 36.1 \\
Exp 2 & 0.9 & 21.4 & 1.6 & 31.8 \\
Exp 3 & 1.3 & 20.1 & 0.0 & 31.2 \\
Exp 4 & 2.8 & 53.0 & 0.0 & 55.3 \\
Exp 5 & 0.0 & 35.5 & 0.1 & 31.1 \\
Exp 6 & 1.3 & 41.4 & 2.6 & 26.0 \\
Exp 7 & 1.9 & 52.6 & 0.2 & 42.3 \\
Exp 8 & 6.4 & 31.3 & 0.4 & 25.3 \\
Exp 9 & 7.8 & 31.2 & 1.1 & 26.3 \\
& & & & \\
average & 2.7 & 36.4 & 0.7 & 33.9 \\
SEM & 0.9 & 3.9 & 0.3 & 3.2 \\
\hline
\end{tabular}

d

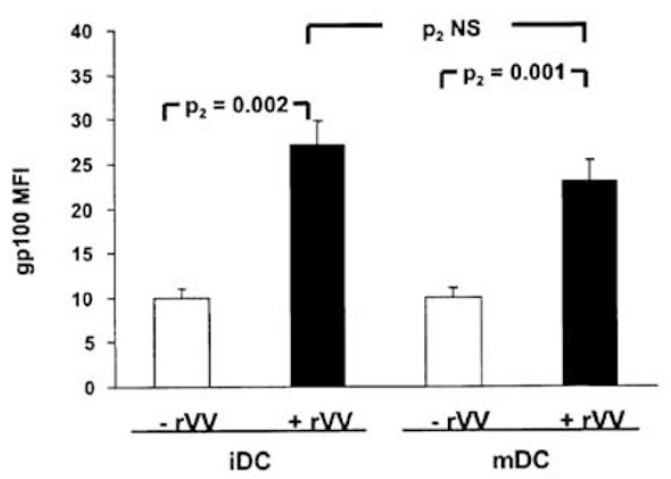

Figure 1 Efficiency of infection and induction of gp100 expression by the $r V V$-gp100 vector. After the maturation step, both iDC and $m D C$ were exposed to 1:10 MOI of $r V V$-gp100 for $1 \mathrm{~h}$ and, after overnight incubation, stained for intracellular expression of gp100 with the $m A b$ HMB45. (a) Histogram of a representative experiment (A375 and SK23 are two melanoma cell lines, one lacking expression of gp100 and the other demonstrating average expression as previously reported $\left.{ }^{63}\right)$. (b) Percent of DC staining with HMB45 (average \pm s.e.m. of nine consecutive experiments). (c) Tabulated variability of the infection efficiency of $r V V$ on iDC versus $m D C$, percentage of gp100-positive cells. (d) Mean fluorescence intensity (MFI) of DC positive for the expression of HMB45 (average \pm s.e.m. of nine consecutive experiments). NS-nonsignificant.
Effect of rVV-gp100 infection on the phenotype of iDC and $m D C$

Since it has been shown that $\mathrm{rVV}$ can inhibit the maturation of $\mathrm{DC}{ }^{24}$ we then tested whether infection by rVV could inhibit the expression of various markers descriptive of the status of maturation of DC. Indeed, it has been shown that exposure to rVV can inhibit the conversion mediated by monocyte-conditioned medium of iDC into mDC. ${ }^{24}$ However, the effect of $\mathrm{rVV}$ infection on the phenotype of already matured DC is not known. Therefore, in six consecutive experiments we tested the expression of HLA-DR, CD80, CD83, and CD86 after exposure of iDC and mDC to 1:10 MOI of $\mathrm{rVV}$ for $1 \mathrm{~h}$, followed by overnight incubation. With the exception of HLA-DR, most surface markers tested demonstrated a trend to downregulation in $\mathrm{rVV}$-infected compared with noninfected DC independent of their status of maturation (Figure 2a). However, only CD83 and CD86 were significantly affected. CD83 MFI decreased from 7.1 \pm 0.6 to $5.6 \pm 0.4$ (paired Student's $t$-test $P_{2}=0.012$ ) in iDC and from $15.0 \pm 1.9$ to $9.5 \pm 1.4$ (paired Student's $t$-test $P_{2}=0.003$ ) in mDC. Similarly, CD86 MFI decreased from $96.3 \pm 25.3$ to $61.7 \pm 13.9$ (paired Student's $t$-test $P_{2}=0.029$ ) in $\mathrm{iDC}$ and from $127.1 \pm 21.9$ to $89.0 \pm 12.3$ (paired Student's $t$-test $P_{2}=0.019$ ) in mDC. Since the expression of HLA-class I alleles, and in particular HLA-A*0201, was especially relevant for the following experiments, in three consecutive experiments we also tested the effect of $\mathrm{rVV}$ infection on HLA-A*0201 expression using the HLA-A2-specific mAb KS-1. ${ }^{26}$ Surface expression of HLA-A ${ }^{*} 0201$ was significantly downregulated by rVV infection and equally affected iDC and $\mathrm{mDC}$ (Figure $2 \mathrm{~b}$ and c). In addition, overnight treatment with IFN- $\alpha$ $(1000 \mathrm{IU} / \mathrm{ml})$ did not restore the expression of HLA$A^{*} 0201$ by either DC population (data not shown). Thus, it appears that $\mathrm{rVV}$ cannot only inhibit the conversion from $\mathrm{iDC}$ to $\mathrm{mDC}$ as described by Engelmayer $e a^{24}$ but it may partially interfere with cellular metabolism in a specific or nonspecific manner by decreasing the expression of maturation markers and other proteins.

\section{Effect of rVV-gp100 infection on HLA-class I-associated antigen presentation}

We previously utilized rVV-infected iDC for the generation of predominantly HLA-A*0201-associated, CD8+ Tcell reactivity toward melanoma Ag. ${ }^{78,27}$ In particular, we observed that this strategy was more efficient than the exogenous pulsing of iDC with saturating levels of relevant epitope. ${ }^{78}$ The reason for the enhanced efficiency remained unclear, and we postulated that the longer persistence of $\mathrm{Ag}$ on the surface of $\mathrm{DC}$ due to the prolonged production of antigenic molecules driven by the viral promoters might have been responsible. ${ }^{8}$ Surprisingly, however, in the conduct of these studies we noted that $\mathrm{rVV}$-infected $\mathrm{DC}$ had very little ability to stimulate generation of IFN- $\gamma$ production by several Agspecific T-cell clones studied (unpublished observation). Since these studies yielded indirect information about TCR engagement as they measured the cumulative production of IFN- $\gamma$ by T-cells over a 24 -h period, we tested the efficiency of $\mathrm{Ag}$ presentation by iDC and $\mathrm{mDC}$ using new technologies that address more directly TCR/ epitope engagement. In particular, we tested the ability of $\mathrm{rVV}$-infected DC to induce TCR downregulation and 
a

HLA DR

CD 80

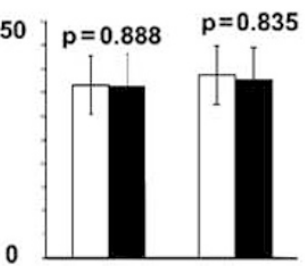

25 $\mathrm{p}=0.351$

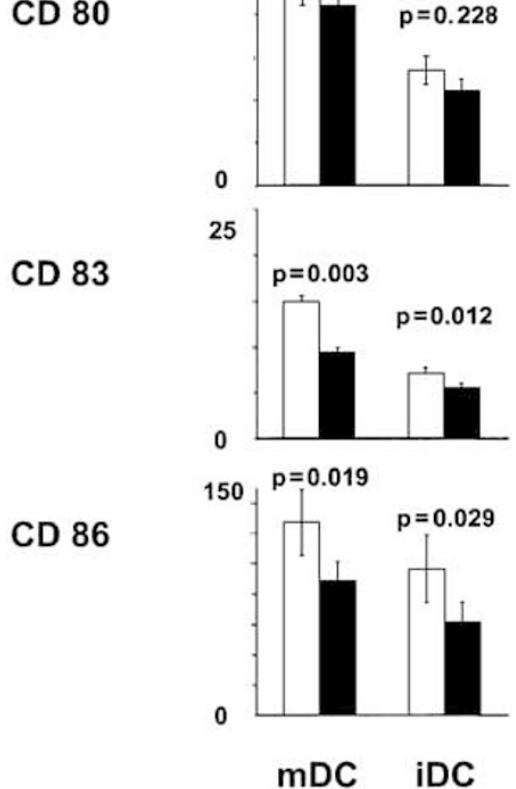

b
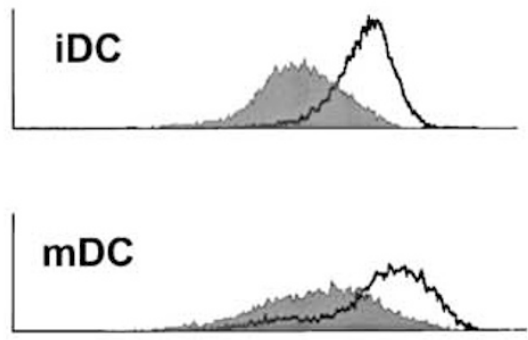

HLA A2 c

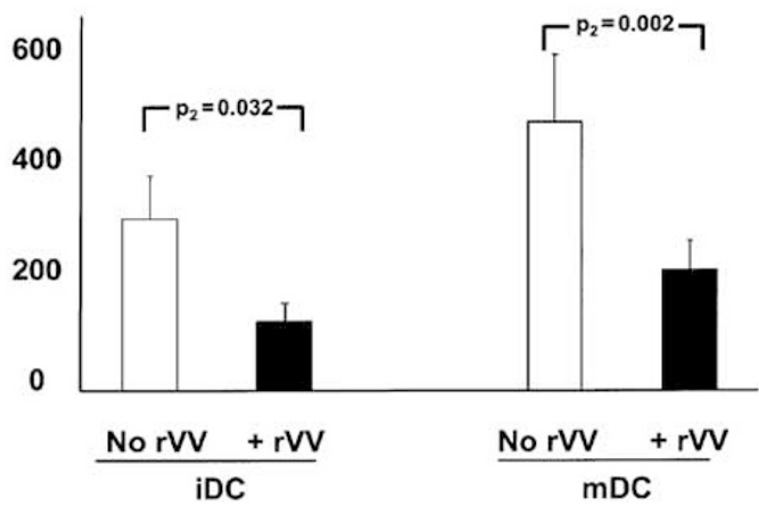

intracellular accumulation of IFN- $\gamma$ in short-term assays as previously described. ${ }^{28,29}$ After infection with $\mathrm{rVV}$ and overnight incubation, the DC were used as antigenpresenting cells to stimulate a well-characterized g209specific, HLA-A*0201-restricted CTL clone JR6C12 representative of most CTL clones derived from HLA$A^{*} 0201$ melanoma patients treated at our institution. In particular, JR6C12 was originally expanded from circulating lymphocytes of a patient who had undergone active-specific immunization with g209-2M peptide administered subcutaneously in incomplete Freund's adjuvant. ${ }^{30}$ JR6C12 was tested for reactivity toward the natural g209 epitope pulsed onto T2 cells (gp100:209-217 at decreasing concentrations from 1 to $0.0001 \mu \mathrm{g} / \mathrm{ml}$ ), the irrelevant gp100:280-288 pulsed onto T2 cells, HLA$\mathrm{A}^{*} 0201$-associated epitope, two HLA-A*0201-negative and two HLA-A*0201-expressing, gp100-expressing melanoma cell lines. After $24 \mathrm{~h}$ of culture, the supernatants were tested for cytokine accumulation (Figure 3). The clone could release discrete amounts of all cytokines tested at g209 concentrations as low as $0.1 \mathrm{ng} / \mathrm{ml}$. This level of responsiveness was previously shown to be associated with good recognition of melanoma cell targets. $^{31}$ Indeed, this concentration of epitope approximated the cytokine release induced by exposure of JR6C12 to HLA-A*0201-expressing melanoma cell lines (bar graphs in Figure 3). In fact, with the exception of IL-2 that was not specifically secreted by JR6C12 upon stimulation with HLA-matched tumor cells, cytokine release was similar to $0.01 \mathrm{ng} / \mathrm{ml}$ g209 stimulation (GMCSF: $1582 \pm 22$ versus $1350 \pm 128$ and $2444 \pm 228$ for g209 versus $526 \mathrm{Mel}$ and $624 \mathrm{Mel}$ stimulated T cells; TNF- $\alpha$ : $45 \pm 7$ versus $66 \pm 7$ and $74 \pm 14$ for g209 versus $526 \mathrm{Mel}$ and $624 \mathrm{Mel}$ stimulated T cells; IFN- $\gamma$ : $3258 \pm 1$ versus $674 \pm 49$ and $2710 \pm 384$ for g209 versus $526 \mathrm{Mel}$ and 624 Mel stimulated T cells cytokine concentrations given are reported as $\mathrm{pg} / \mathrm{ml}$ ). None of the non-HLA-A*0201matched targets induced cytokine release as well as the irrelevant gp100:280-288 HLA-A*0201-associated epitope at the concentration of $1 \mu \mathrm{g} / \mathrm{ml}$. Thus, this CTL clone fits the profile of a high avidity $\mathrm{T}$ cell that can recognize the naturally processed and presented g209 epitope.

In five consecutive experiments, JR6C12 was cocultured for $6 \mathrm{~h}$ at a 3:1 effector:stimulator ratio with the melanoma cell lines A375 and SK23, respectively, lacking and expressing gp100. In addition, JR6C12 was exposed to iDC and mDC previously infected with $\mathrm{rVV}$ encoding gp100. There was no significant difference in the expression of gp100 by rVV-infected iDC and mDC compared with SK23, while the expression of this Ag was significantly increased in these cells compared with $\mathrm{A} 375$ and noninfected iDC and $\mathrm{mDC}$ (t-test $P_{2}$ value $<0.01$ in all circumstances). The expression of

Figure 2 Effect of $r V V$ infection on the status of maturation of $i D C$ and $m D C$. (a) After 1 h exposure to $r V V$ (1:10 MOI), DC were maintained in culture overnight and then cells were stained for HLA-DR, CD80, CD83, and CD86. The average \pm s.e.m. of the mean fluorescence intensity (MFI) for each marker is presented here for six consecutive experiments. Noninfected $D C$ are shown as white bars while $r V V$-exposed $D C$ are shown as black. (b) Representative histogram of surface HLA-A2 expression in $i D C$ and $m D C$ exposed (dark histogram) or not exposed (white) to $r V V$ infection. (c) Average MFI in six consecutive experiments (and associated s.e.m.) of HLA-A2 expression in iDC and $m D C$ in the presence or absence of $r V V$ infection (all $\mathrm{P}$-values represent the statistical analysis of a two-tailed paired $t$-test). 
gp100 by iDC and mDC was not sufficient to induce detectable levels of IFN- $\gamma$ expression by JR6C12, while a modest but significant percentage of $\mathrm{T}$ cells $(5.3 \pm 0.8)$ expressed IFN- $\gamma$ upon exposure to the gp100-expressing SK23 (Figure 4a). TCR downregulation appeared to be a more sensitive marker of Ag-specific T-cell engagement
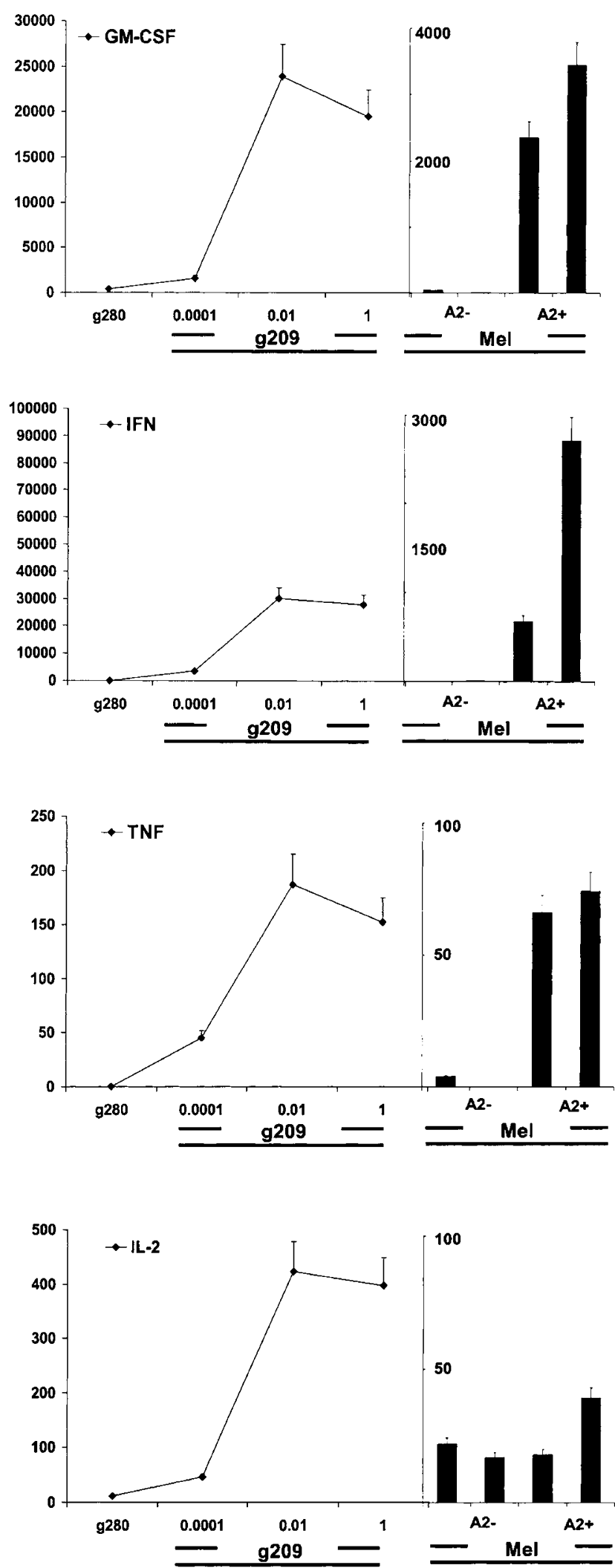

since we could observe nearly $31.2 \pm 6.8 \%$ decrease in MFI in cells stimulated with SK23 and a lesser but detectable decrease upon stimulation with iDC (12.8 \pm 4 , $t$-test $P_{2}$ value $=0.02$ compared with SK23) and $\mathrm{mDC}$ $\left(18.5 \pm 5.2, t\right.$-test $P_{2}$ value $=0.07$ compared with $\left.S K 23\right)$ infected with rVV (Figure 4b). In addition, all T-cells demonstrated a certain reduction of TCR expression as the fluorescence intensity was homogeneously shifted to lower levels in SK23-, iDC-, and mDC-stimulated T cells.

To exclude that HLA-A*0201 downregulation on rVVinfected DC could influence the presentation of relevant epitope, JR6C12 was also exposed to DC pulsed with limiting amounts of g209-2M characterized by high binding affinity for this HLA allele. ${ }^{13}$ JR6C12 was exposed to iDC, iDC + rVV-gp100, mDC, and $\mathrm{mDC}+$ rVV-gp100. The effect of such exposure was analyzed on intracellular accumulation of IFN- $\gamma$ (Figure 4, panel c) and TCR downregulation (percent decrease in TCR MFI compared with non-stimulated JR6C12, Figure 4, panel d). JR6C12 could recognize g209-2M pulsed DC at peptide concentrations as low as $0.01 \mathrm{ng} / \mathrm{ml}$ as judged by TCR downregulation. However, peptide concentrations of $1 \mathrm{ng} / \mathrm{ml}$ were necessary to detect any evidence of IFN- $\gamma$ accumulation in a few T cells and $100 \mathrm{ng} / \mathrm{ml}$ to identify IFN- $\gamma$ accumulation in the majority of T-cells. Thus, TCR downregulation was the most sensitive marker of TCR/epitope engagement. This finding might explain our previous observation that the percent of Tcells staining with tetrameric HLA-peptide complexes and demonstrating TCR downregulation upon cognate stimulation was consistently higher than the number of intracellular IFN- $\gamma$-expressing $\mathrm{T}$ cells. ${ }^{28,32}$ In addition, these data suggest that the ability of DC to present Ag is not significantly limited by the decreased surface density of HLA-A*0201 molecules occurring 12-24 h after rVV-gp100 infection.

\section{Endogenous processing and presentation of rVV-Gp100 to HLA-class l-restricted CD8+ T cells}

In five consecutive experiments, the ability of iDC and $\mathrm{mDC}$ to process and present endogenous or exogenous Ag was compared by stimulating JR6C12 with infected and noninfected DC and the two melanoma cell lines (Figure 5a). In all conditions, a parallel experiment was conducted in which the stimulator cells had been previously loaded with $10 \mu \mathrm{g} / \mathrm{ml}$ of g209-2M to test the intrinsic capability of DC to present HLA-class I-associated epitopes independent of their ability to process endogenous Ag. In this set of experiments, it appeared that the intrinsic ability of DC to present exogenously provided epitope was not affected by their status of maturation or by their exposure to rVV-gp100.

Figure 3 Characterization of the gp100-specific CD8+ T-cell clone JR6C12. The CD8+CTL clone JR6C12 was originally expanded from circulating lymphocytes of a patient who had undergone active specific immunization with g209-2M peptide administered subcutaneously in incomplete Freund's adjuvant. ${ }^{30}$ This clone was stimulated for reactivity toward the natural g209 epitope (gp100:209-217 at decreasing concentrations from 1 to $0.0001 \mu \mathrm{g} / \mathrm{ml})$, with the irrelevant gp100:280-288 (1 $\mu \mathrm{g} /$ $m l), H L A-A^{*} 0201$-associated epitope, and with two HLA- $A^{*} 0201-n e g a t i v e$ and two HLA-A*0201-expressing, gp100-expressing melanoma cell lines. After $24 \mathrm{~h}$ of culture, the supernatants were tested for cytokine accumulation. Data represent the average \pm s.e.m. of three experiments and represent $\mathrm{pg} / \mathrm{ml}$ of cytokine in the supernatant. 
However, their ability to process and present endogenous Ag appeared to be quite limited independent of their status of maturation. Also in this set of experiments, TCR downregulation appeared to be a more sensitive marker

a

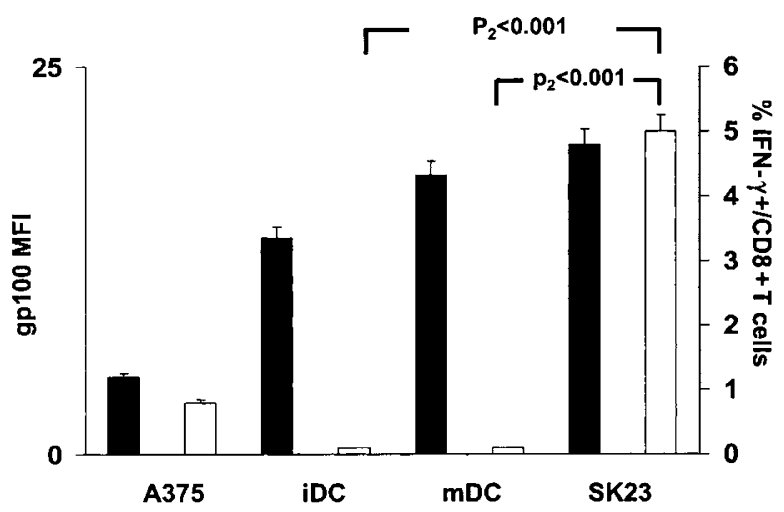

b



C



d

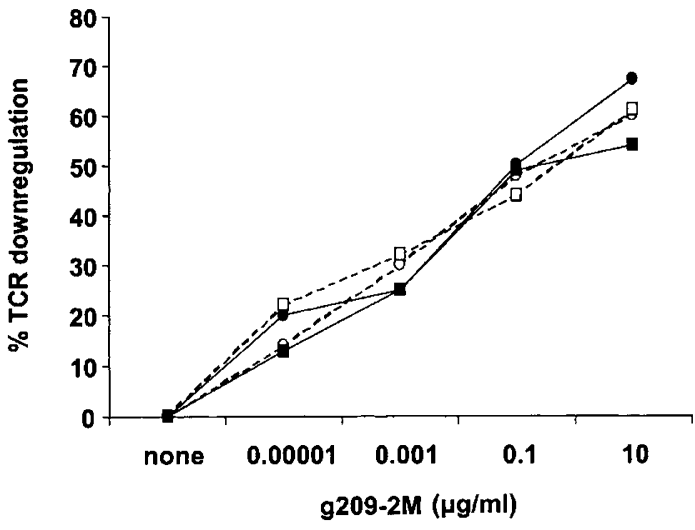

of T-cell epitope engagement than intracellular IFN- $\gamma$ accumulation.

Based on these findings, it was not clear whether the effectiveness of $\mathrm{Ag}$ processing and presentation was dampened by rVV infection or if the biochemical properties of the epitope involved in T-cell activation bore the most significant impact. Therefore, we repeated the same set of experiments by stimulating JR6C12 with DC that had been previously infected with an rVV containing a modified gp100 construct to express 209-2M rather than the wild-type 209 epitope (Figure 5b). This caused a dramatic change in the ability of DC to present epitope to JR6C12 independent of their status of maturation. In both cases, $\mathrm{rVV}$-infected DC could induce the same amount of TCR downregulation and IFN- $\gamma$ expressing JR6C12 as DC that had been pulsed with saturating amounts of g209-2M $(10 \mu \mathrm{g} / \mathrm{ml})$. From this set of experiments, it appears that DC retain the capability to process and present $\mathrm{Ag}$ in the context of rVV infection. However, the efficiency of Ag presentation is highly dependent on the specificity of the HLA-epitope interaction. These findings may explain our previous observation of the stringent allele-epitope specificity of MART-1/MelanA immune dominance. ${ }^{27}$ To exclude Tcell clonotype-specific preferences in the recognition of the mutated versus wild-type epitope. The critical experiment described in Figure $5 b$ was reproduced utilizing two additional g209 clones (CK3H6 and S6G3) expanded from bulk populations from other individuals affected by melanoma: the T-cell clones were stimulated with rVV-infected as well as g209-2M-pulsed mDC (Figure 6). A very similar pattern of IFN- $\gamma$ expression (Figure 6a) as well as TCR downregulation (Figure 6b) was also noted in these two clones. In addition, identical results were noted using rVV-infected and peptidepulsed iDC (data not shown).

\section{Endogenous processing and presentation of rVV-Gp100 to HLA-class II-restricted CD4+ T cells}

A major advantage of whole $\mathrm{Ag}$ delivery through recombinant vectors is the theoretical possibility of delivering several epitopic determinants simultaneously. This could be of particular advantage if HLA-class II-associated epitopes could be presented in combination with HLA-class I-associated epitopes to sustain activation

Figure 4 Effect of the coculture of $r V V$-infected DC with the gp100specific CD8+ T-cell clone JR6C12. The CD8+ T-cell clone JR6C12 was cocultured at a 3:1 effector:stimulator ratio for $6 \mathrm{~h}$ with the melanoma cell lines A375 and SK23, respectively, lacking and expressing the melanoma antigen gp100. In addition, the same clone was exposed to iDC and mDC that had been infected previously with $r V V$ encoding gp100. The black bars represent the expression of gp100 by the melanoma cell lines and the infected DC (gp100 expression was measured only in the gated population of DC that demonstrated virally driven expression; see Figure $3 a$ ). The white bars show the percent of CD8+ T cells staining for intracellular content of IFN- $\gamma$ (panel (a)) or the percent decrease in MFI for TCR staining (panel (b)) following exposure to the various stimulator cells. Data represent the average \pm s.e.m. of five consecutive experiments. To exclude the possibility that downregulation of $H L A-A^{*} 0201$ itself could affect the presentation of relevant epitope, JR6C12 was also exposed to DC pulsed with limiting amounts of the gp100:209-217(210M) peptide (209$2 M)$ characterized by high binding affinity of this HLA allele. ${ }^{13}$ The T-cell clone was exposed to iDC $(\bigcirc), i D C+r V V(\bullet), m D C(\square)$, and $m D C+r V V$ (ם). The effect of such exposure was analyzed on IFN- $\gamma$ intracellular accumulation (panel c) and on TCR downregulation (percent decrease in TCR MFI compared with nonstimulated JR6C12, panel (d)). 


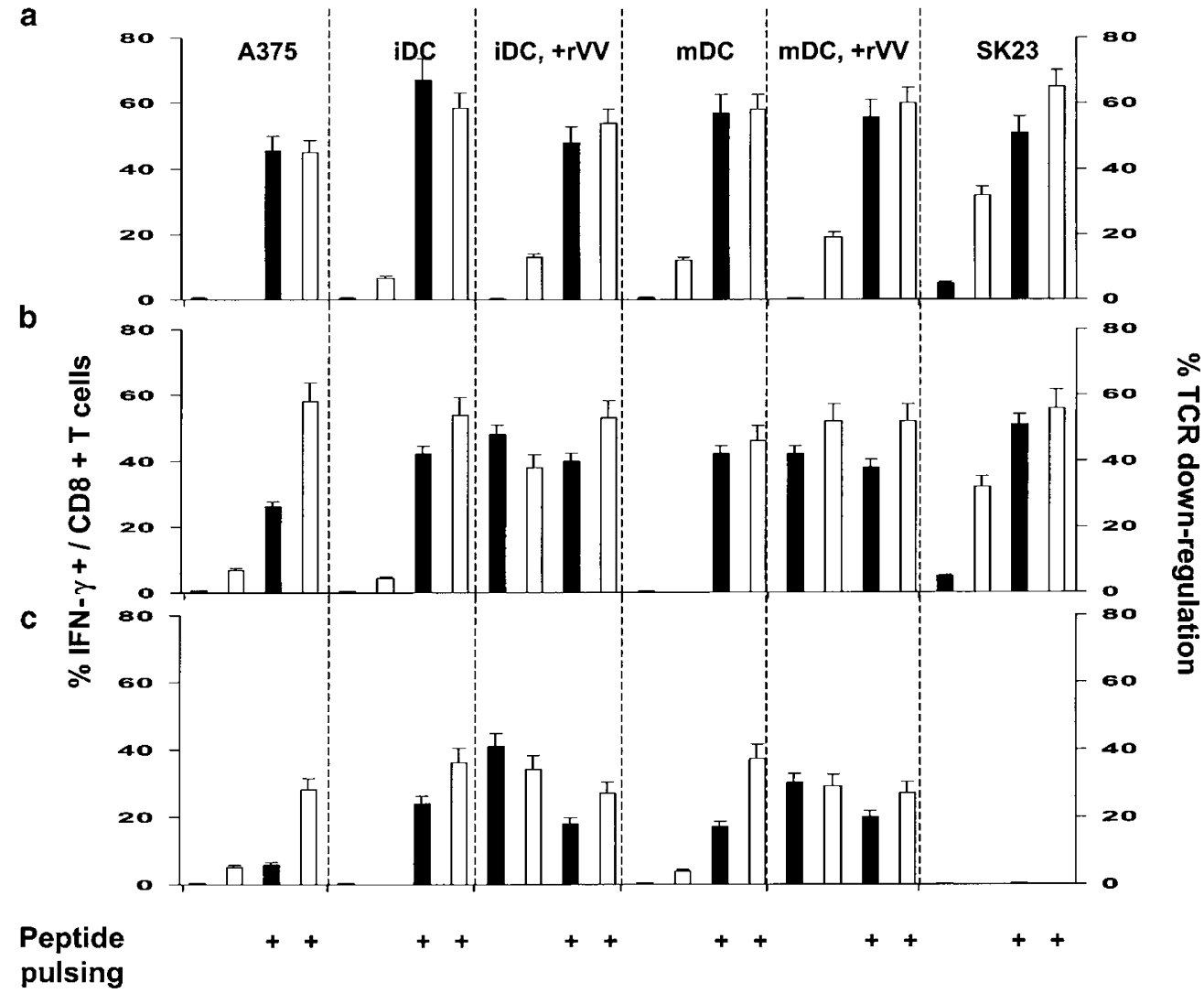

Figure 5 Ability of DC to process and present endogenously provided Ag. T-cell clones were stimulated with iDC or $m D C$ that had been previously infected or noninfected with $r V V$ and had or had not received exogenous pulsing with peptide. As a control, the T-cell clones were stimulated with the HLA-A*0201-, DRB1*0701-expressing gp100-negative cell line A375 (HLA- $\left.A^{*} 0101,0201, D R-\beta 1^{*} 0405,0701\right)$ and the HLA- $A^{*} 0201$-expressing gp100-positive cell line SK23 (HLA- $\left.A^{*} 0101,0201, D R-\beta 1^{*} 0101,1301\right)$. Effect on IFN- $\gamma$ accumulation in responding T cells is presented by the black bars as average \pm s.e.m. of the percent of T cell expressing detectable amount of IFN- $\gamma$ in five consecutive experiments in panel a and in three consecutive experiments in panels (b and $c)$. White bars present the effect on TCR downregulation as average \pm s.e.m. of the percent reduction in MFI of TCR staining compared with nonstimulated T cells. (a) The g209-specific, HLA-A*0201-restricted CD8+ T-cell clone JR6C12 was stimulated with HLA-A*0201-expressing DC infected with rVV encoding wild-type gp100. (b) JR6C12 was stimulated with HLA-A*0201-expressing DC infected with $r V V$ encoding a gp100 gene with a mutation to induce expression of the 209-2M epitope. (c) The HLA-DR $\beta 1^{*} 0701$-restricted, gp100:170-190-specific CD4+ T-cell clone (F11) was stimulated with HLA$D R \beta 1^{*} 0701$, expressing DC infected with $r V V$ encoding wild-type gp100. The peptide used for DC loading was gp100:209-2M for experiments shown in panels (a) and (b) and gp100:170-190 for experiments shown in panel (c). The gp100:209-2M peptide was used at 10 mM, the peptide gp100:170-190 was used at $50 \mathrm{~mm}$.

of APC and other immune cells..$^{33,34}$ Thus, we investigated, in three consecutive experiments, the ability of iDC and $\mathrm{mDC}$ to induce activation of an HLADR $31^{*} 0701$-restricted CD4+ T-cell clone (F11) upon infection with rVV-gp100 and/or exogenous pulsing with the relevant epitope gp100:170-190 (LSIGTGRAMLGTHTMEVTVYH). ${ }^{35}$ In this set of experiments, no differences were noted between the ability of iDC and $\mathrm{mDC}$ to induce TCR downregulation and IFN- $\gamma$ production by F11 upon rVV infection (Figure 5c). In addition, it appeared that the transgene could induce surface epitope expression comparable to those obtainable in saturating conditions of exogenous epitope delivery, since no differences were noted in T-cell activation in response to peptide-pulsed compared with rVV-infected iDC or mDC. Thus, endogenous processing of HLA-class IIassociated epitopes is similar between iDC and $\mathrm{MDC}$ and it is not altered by rVV infection.

\section{Effect of rVV infection on cytokine production by iDC and $m D C$}

We investigated whether rVV infection had functional repercussions on the cytokine profile of iDC and $\mathrm{mDC}$ by testing in four consecutive experiments supernatants from DC cultures exposed or not exposed to rVV infection using a protein chip array (Figure 7). In general, in the experimental conditions tested, rVV infection had no effect on the ability of DC to release cytokines, although a trend was noted in an overall decrease in cytokine accumulation in the presence of $\mathrm{rVV}$ infection. This trend reached statistical significance only in the case of IL-12, whose expression was reduced in rVV-infected $\mathrm{mDC}$.

\section{Discussion}

Several groups have repeatedly shown that immunization with minimal epitopic determinants is very effective in inducing HLA-class I-associated CD8+ T-cell responses. ${ }^{36}$ By restricting the immunogen to a single epitope/HLA allele combination, this approach is, however, intrinsically limited by the prevalence of a given HLA allele in the population to be treated ${ }^{37}$ and by the level of expression of such an allele by tumor cells, which may vary in natural conditions or in adjustment to 
a

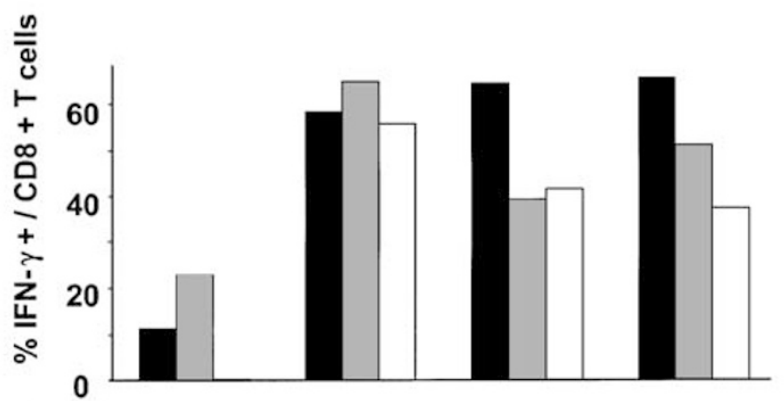

b

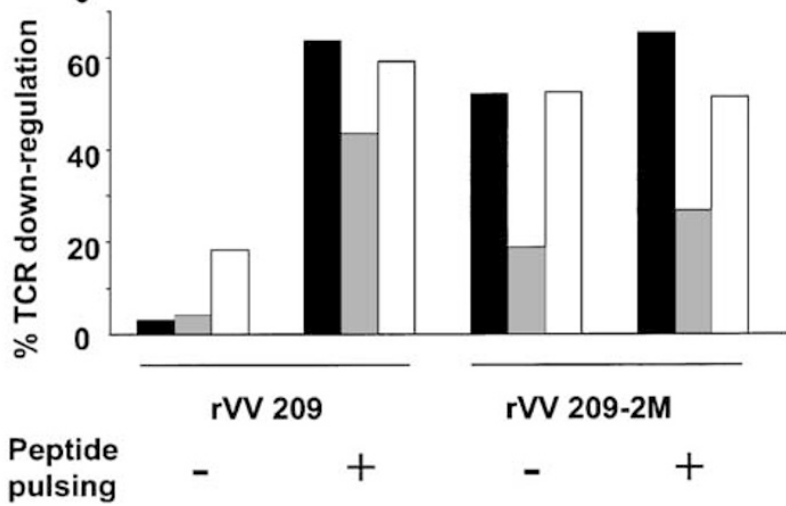

Figure 6 Presentation and recognition of endogenously processed $\mathrm{Ag}$ is independent of T-cell clonotypic differences. The gp100:209-217-specific Tcell clones CK3H6 (black bars), S6G3 (gray bars), and JR6C12 (white bars) were exposed to $m D C$ infected or noninfected with $r V V$ and had or had not received exogenous pulsing with peptide. Intracellular IFN- $\gamma$ expression (a) and TCR downregulation (b) are shown. Similar results were obtained with iDC (not shown).

the immune pressure induced by the immunogen. ${ }^{38}$ Therefore, effective expression of whole Ag would be more desirable based on the assumption that $\mathrm{Ag}$ processing and presentation by the host cells could naturally tailor the antigenic potential to the HLA phenotype of individual patients to induce broader immune responses less susceptible to HLA allele or haplotype-specific loss. ${ }^{39}$

Thus, various strategies have been suggested to cause expression of whole Ag in vivo with the purpose of priming polyvalent CD8+ T-cell/HLA-class I-restricted and CD4+ T-cell /HLA-class II-restricted immune responses. These strategies include administration of expression vectors, ${ }^{40,41}$ naked DNA, ${ }^{42,43}$ chaperon delivery systems such as autologous heat-shock proteins, ${ }^{44}$ pools of unselected protein extracts such as tumor lysates, ${ }^{45}$ or whole proteins. ${ }^{46}$ An appealing tactic for $\mathrm{Ag}$ delivery includes the induction of $\mathrm{Ag}$ expression in DC to combine the pluripotentiality of whole Ag delivery with the costimulatory properties of these professional antigen-presenting cells., $77-50$ The success of these strategies is based on the assumption that the chances of a given molecule to be immunogenic are relatively uniformly distributed across the HLA polymorphism.

It has also been suggested that infection of DC with viral expression vectors to induce $\mathrm{Ag}$ expression may have the additional benefit of promoting activation of these cells through a danger signal intrinsic to the biology of the viral infection. ${ }^{34}$ Indeed, we have utilized in the past $\mathrm{rVV}$ for the in vitro induction of antimelanoma differentiation antigen-specific CTL.7,8 We, however,
IL-2

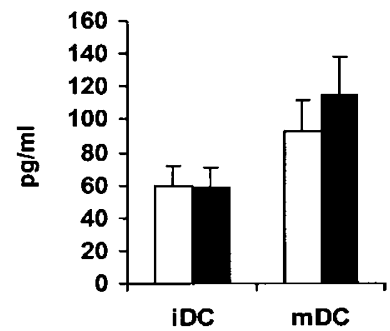

IL-12

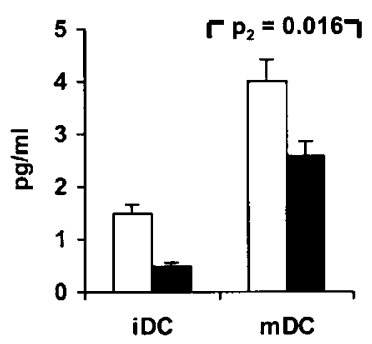

IL-10

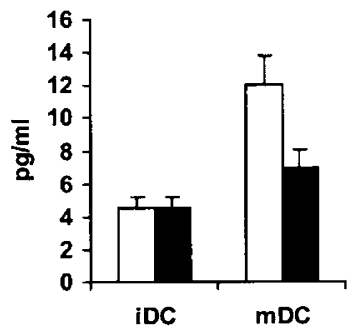

TNF- $\alpha$

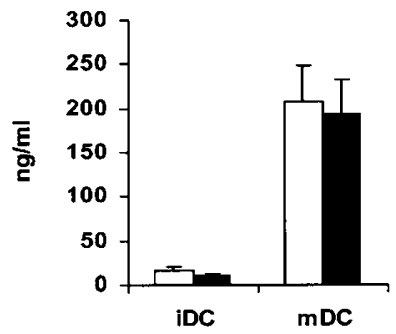

Figure 7 Profile of selected cytokines upon infection of $i D C$ and $m D C$ with $r V V$. Supernatants of DC exposed (black bars) or nonexposed (white bars) to $r V V$-gp100 were tested on a protein chip (Search Light Proteome Array $\left.^{\mathrm{TM}}\right)$. Data are presented only for the most interesting cytokines. Results represent the average \pm s.e.m. of four consecutive experiments. With the exception of IL-12, paired t-test $P_{2}$ values comparing infected versus non-infected $D C$ (whether mature or immature) were all nonsignificant and are not shown. Of note, IL-10 is also not significantly reduced postinfection in $\mathrm{mDC}(P=0.117)$.

noted that MART-1/MelanA-specific immune reactivity was more easily elicited than that of the larger melanoma differentiation antigen gp100. In addition, we observed that the reactivity elicited against MART-1 was almost exclusively limited to the HLA-A*0201-associated MART-1:27-35 epitope, suggesting that the immunogenicity of a protein may not be necessarily expandable to multiple HLA alleles as desired by the whole Ag vaccination approach. Although others have reported successful induction of gp100-specific T cells using rVVinfected DC, ${ }^{48,49}$ our parallel studies where induction of MART-1 reactivity was compared with that of gp100 using identical vectors demonstrated a much lower effectiveness of the gp100 construct to induce Ag-specific $\mathrm{T}$ cells in spite of identical efficiency of infection and expression of Ag (unpublished data).

Nevertheless, it appeared in parallel experiments that rVV-based delivery of MART-1/MelanA through DC in vitro was more efficient in inducing CD8+/HLA-A*0201associated immune responses against MART-1:27-35 than exogenous pulsing of DC with saturating levels of the same epitope. ${ }^{7}$ Due to the suggested influence of the duration and intensity of Ag stimulation on the fate of T-cell activation, ${ }^{19,20}$ we originally postulated that the higher immunogenicity of rVV-induced Ag delivery was due to prolonged expression of MART-1 driven by rVV infection compared with the short permanence of the same epitope after exogenous pulsing. ${ }^{8}$ Others, however, have convincingly demonstrated that rVV infection may have a detrimental role on the function of DC by interfering with their maturation 
and, as a consequence, efficiency of Ag presentation. ${ }^{24}$ Thus, it remains questionable how rVV deliver Agspecific stimulation in the context of active-specific immunization.

Since DC maturation is a step that can be achieved in vitro before adoptive transfer for therapeutic purposes, ${ }^{51}$ we first assessed the sensitivity of iDC and $\mathrm{mDC}$ to $\mathrm{rVV}$ infection. In addition, we tested whether rVV infection could alter the status of activation and/or differentiation of iDC and mDC. Overall, in the experimental conditions tested, rVV could infect and induce expression of gp100 with similar efficiency in iDC and mDC. In addition, the effect of the viral infection was relatively minor in both types of cells at the time point evaluated. In general, a depression of most cellular functions was noted with a trend to decrease surface expression of HLA-class I alleles and costimulatory molecules and reduce release of cytokines, suggesting a nonspecific depression of the metabolic status of the cell associated with the viral infection. To assess whether rVV infection could affect the ability of DC to present endogenously processed Ag, we tested the ability of $\mathrm{rVV}$-infected iDC and $\mathrm{mDC}$ to induce TCR downregulation as well as induce production of IFN- $\gamma$. These assays were selected over other methods to assess Ag-specific stimulation of T-cells because they appear to provide the most direct and sensitive evidence of TCR/epitope engagement. In addition, we and others have previously shown that they correlate quite well with other methods of assessing Ag-specific stimulation. ${ }^{16,28,29,32,52}$ Because of the significant reduction in HLA-A*0201 expression caused by rVV infection, we first tested the ability of rVV-infected DC to induce TCR downregulation and IFN- $\gamma$ expression in the g209-specific CD8+ T-cell clone JR6C12 when exogenously pulsed with gradually decreasing concentrations of relevant epitope. No significant differences were noted in the ability of iDC or $\mathrm{mDC}$ to induce T-cell activation, whether they had been infected or not, suggesting that the cell surface availability of functional HLA-A*0201 molecules in the conditions tested was not a major limiting factor.

Interestingly, however, virally driven expression of gp100 could not induce IFN- $\gamma$ expression and caused only minimal TCR downregulation in spite of levels of gp100 expression comparable to those of a melanoma cell line capable of inducing a moderate stimulation of the same T-cell clone. To test whether this lack of stimulation was due to Ag processing, trafficking or presentation defects in DC upon rVV infection or to a low affinity of the natural g209 epitope for HLA-A*0201 that could affect its endogenous presentation, we infected DC with rVV encoding a gp100 construct containing the g209-2M modification. Since this restored the ability of DC to induce stimulation of JR6C12, we conclude that rVVinfected iDC or $\mathrm{mDC}$ maintain their ability to process and present endogenous Ag and that their Ag-presentation efficiency is mainly dependent on the biochemical properties of a given peptide. It is possible that the higher binding affinity of g209-2M for HLA-A*0201 is the sole explanation for this phenomenon. ${ }^{13}$ Interestingly, however, this biochemical property appears to be more significant in the context of endogenous presentation, since in titration experiments the differential response of JR6C12 to either peptide provided exogenously was relatively narrow.
This study shows that DC are not particularly efficient in inducing HLA-class I-associated Ag-specific stimulation of CD8+ T cells unless a strongly binding peptide is used. This, of course, is not surprising since their function is rather dependent on the costimulatory function of various cell surface molecules or the immune stimulatory and chemoattractant properties of released cytokines. ${ }^{51,53}$ Contrary to other viral systems, ${ }^{54}$ these functions appear only minimally affected in the early phases of rVV infection, when DC are still viable. Furthermore, both iDC and mDC appear very efficient in presenting endogenous Ag to HLA-class II-restricted CD4+ $\mathrm{T}$ cells. It is also likely that part of the Ag presentation occurring in vivo could be dependent upon endogenous scavenger DC that, upon virally induced cell death at the vaccination site or in draining lymph nodes, can cross-present rVV-derived Ag.,55,56 Although rVV infection has been shown to impede maturation of $\mathrm{DC}_{1}{ }^{24}$ it does not appear, according to our study, to rapidly revert the function and properties of DC after completion of the maturation process. In fact, rVV infection only minimally affected the status of maturation of DC in the experimental conditions tested here. This point may be particularly important since DC maturation is a requirement for efficient activation of CD8+ $\mathrm{T}$ cells. ${ }^{21}$ Importantly, DC maturation is not associated with decreased efficiency of transgene expression. Thus, rVV infection should follow the maturation step and rVV-infected mDC could be a useful tool for immunization, given the presence of an appropriate epitope/allele combination in the selected Ag.

The importance, however, of Ag-specific stimulation cannot be underestimated by assuming ad hoc immunogenicity across the HLA polymorphism. ${ }^{37}$ Experience has shown that there is a discrepancy between the number of peptides within an Ag that can be predicted to bind to various HLA alleles and the number of epitopes actually recognized in a CTL response to the same protein. ${ }^{27,57,58}$ Consequently, the 'dominant' peptides that can function as antigenic determinants are far fewer than the potential HLA binders, while most peptide sequences have 'cryptic' immunogenic properties. As we have previously shown, efficient presentation of immunogenic/ 'dominant' epitopes is dependent upon stringent and often unpredictable pairing of an HLA allele with a given peptide. Even well-characterized immunogenic epitopes derived from self-proteins may have relatively cryptic immunogenicity compared with their heteroclitic analogs when priming predominantly naïve immune responses, as exemplified by MART-1/Melan $A^{59,60}$ and, in this case, gp100. Thus, it appears that whole Ag immunization by adoptive transfer of rVV-infected $\mathrm{mDC}$ may not necessarily induce the broad immune responses predicted by the potential of peptide binding to the HLA allelic repertoire of a given patient. Instead, antigenic constructs should be carefully designed to match the known immunogenic potential of a protein with appropriate HLA phenotypes. Since the prevalence of 'dominant' epitopes in nature is probably lower than theoretically predictable by HLA-binding algorithms, it might be more logical to design expression vectors based on available information about the immunogenicity of given HLA/peptide combinations. For instance, some have suggested the development of polyepitope constructs spanning several Ag. ${ }^{50}$ This strategy may prove 
more successful in inducing systemic T-cell responses than relying on the fortuitous occurrence of dominant HLA/epitope combinations.

\section{Materials and methods}

\section{Culture medium}

All cells were maintained in complete medium (CM) consisting of Iscove's medium (Biofluids, Rockville, MD, USA) supplemented with $10 \%$ heat-inactivated human $\mathrm{AB}$ serum (Gemini Bioproducts, Inc., Calabasas, CA, USA), $10 \mathrm{mM}$ HEPES buffer (Cellgro, Mediatech, Inc., Herndon, VA, USA), $0.03 \%$ L-glutamine (Biofluids), $100 \mathrm{U} / \mathrm{ml}$ penicillin/streptomycin (Biofluids), $10 \mu \mathrm{g} / \mathrm{ml}$ ciprofloxacin (Bayer, West Haven, CT, USA), and $0.5 \mathrm{mg}$ / $\mathrm{ml}$ amphotericin B (Biofluids).

Dendritic cells, melanoma cell lines, and T-cell clones PBMC from HLA-A*0201-positive or HLA-DR $\beta 1^{*} 0701$ positive healthy donors recruited by the Department of Transfusion Medicine, NIH, were isolated by Ficoll gradient separation and frozen in human $A B$ serum supplemented with $10 \%$ DMSO and stored in liquid nitrogen until analysis. PBMC were processed for preparation of DC as previously described. ${ }^{61}$ In brief, after thawing, PBMC were cultured at a concentration of $5 \times 10^{7} / \mathrm{ml}$ in $20 \mathrm{ml} \mathrm{CM}$ in $75 \mathrm{~cm}^{2}$ tissue culture flasks (Costar, Cambridge, MA, USA) for $3 \mathrm{~h}$ at $37^{\circ} \mathrm{C}$. Then, nonadherent cells were removed by gentle aspiration with a $10 \mathrm{ml}$ pipette (Costar), while the remaining adherent cells were washed twice with PBS and cultured for 5 days in $\mathrm{CM}$ supplemented with human recombinant GM-CSF (Pepro Tech, Inc., Rocky Hill, NJ, USA) $\left(10^{3} \mathrm{U} / \mathrm{ml}\right)$ and human recombinant interleukin-4 (IL-4) $\left(10^{3} \mathrm{U} / \mathrm{ml}\right.$, Pepro Tech). These two cytokines were added on day 0,2 , and 4 . On day 5, CD40L (Pepro Tech, final concentration $500 \mathrm{ng} / \mathrm{ml}$ ) and LPS (Sigma, St Louis, MO, USA, final concentration $5 \mu \mathrm{g} / \mathrm{ml}$ ) were added to half of the DC to induce maturation. ${ }^{62}$ On day $7, \mathrm{DC}$ were harvested for use. The HLA-A*0201-expressing melanoma cell lines A375-MEL (ATCC CRL 1619) and SK23MEL (ATCC HTB 71) were purchased from ATCC (Rockville, MD, USA). A375 is a cell line not expressing gp100, whereas SK23 is positive for gp100 expression. JR6C12 is a CD3+ CD8+ T-cell clone characterized for reactivity against gp100:209-217 (IMDQVPFSV, g209) as well as against the modified gp100:209-217(210M) (IMDQVPFSV, g209-2M) as previously described (patient \# 10 in Dudley et al ${ }^{30}$ ). JR6C12 testing for epitope specificity was performed by incubation with T2 cells pulsed with the natural g209 peptide epitope at decreasing concentrations $(1-0.0001 \mu \mathrm{g} / \mathrm{ml})$, the irrelevant gp100:280-288 (1 $\mu \mathrm{g} / \mathrm{ml})$, HLA-A*0201-associated epitope, with two HLA-A*0201-negative and two HLA$A^{*} 0201$-positive, gp100-expressing melanoma cell lines. After $24 \mathrm{~h}$ of culture, the supernatants were tested for cytokine accumulation using ELISA kits (Endogen). F11 is a CD3+ CD4+ T-cell clone specific for gp100:174-190 (HLA-DR $\beta 1^{*} 0701$-restricted) epitope TGRAMLGTHTMEVTVYH. ${ }^{35}$ S6G3 and CK3H6 are two further CD3+ CD8+ T-cell clones characterized for reactivity against gp100:209-217 (IMDQVPFSV, g209) as well as against the modified gp100:209-217(210M). All T-cell clones were thawed the day before stimulation and rested overnight

\section{Vaccinia viruses and infection of $D C$}

Recombinant vaccinia- viruses containing native human gp100 (rVV-209) or modified human gp100 (rVV-209$2 \mathrm{M})$, respectively, were provided by Therion Biologics (Cambridge, MA, USA). Infection was performed by centrifuging $3 \times 10^{6} \mathrm{DC}$ in a $15 \mathrm{ml}$ tube (Sarstedt, Newton, NC, USA) in CM, gentle removal of supernatant and resuspension in a solution of $50 \mu \mathrm{l}$ PBS containing $3 \times 10^{7}$ PFU of $\mathrm{rVV}$ (MOI 1:10). The cells were then centrifuged $(500 \mathrm{rpm}, 2 \mathrm{~min})$, rested for $1 \mathrm{~h}$ at $37^{\circ} \mathrm{C}$ shaking the tubes every 15 mins. After $1 \mathrm{~h}, \mathrm{CM}$ was added, the cells were washed and rested overnight in an incubator.

\section{Cytokine profiling of DC supernatants}

At $24 \mathrm{~h}$ after rVV infection, a set of supernatants (four infected and four paired noninfected iDC and $\mathrm{mDC}$ ) was collected. The supernatants were analyzed for the expression of a panel of cytokines using Th1/Th2 protein chip arrays (Search Light Proteome Array ${ }^{\mathrm{TM}}$, Perbio, Boston, MA, USA).

\section{FACS analysis of maturation state and gp100 expression of $D C$}

Cell surface expression level of various markers and gp100 expression by DC was determined by flow cytometry. Washed DC were suspended at a concentration of $2 \times 10^{5}$ cells per $50 \mu$ l FACS buffer (phosphate buffer plus 5\% FCS; Biofluids). DC were incubated at $4{ }^{\circ} \mathrm{C}$ in the dark for $30 \mathrm{~min}$ with $10 \mu \mathrm{l}$ of the respective fluorochrome-labeled antibodies. Then, cells were washed in $4 \mathrm{ml}$ of FACS buffer and analyzed using a FACScalibur flow cytometer and CellQuest software (Becton Dickinson). At least 30000 events were acquired for analysis. The antibodies used were anti-CD80-FITC, anti-CD83-FITC, anti-CD86-FITC, anti-HLA-DR-FITC, KS-1 (anti-HLA-A2), and HMB (anti-gp100). Except for KS-1 ${ }^{26}$ and HMB45 (Enzo Diagnostics, Farmingdale, NJ, USA), all antibodies were purchased from Becton Dickinson. For analysis of gp100 expression, cells were fixed in acetone for $5 \mathrm{~min}$ and then washed prior to staining. Rat-anti-mouse-IgG1-FITC was used as secondary antibody for KS-1 anti-HLA-A2 and HMB anti-gp100 antibodies after a further washing step. Staining data were reported as percentage of positive cells or as MFI.

\section{Peptide pulsing}

The peptides g209 and g209-2M were purchased from Princeton Biomolecules, (Columbus, OH, USA). The peptide gp100:170-190 (LSIGTGRAMLGTHTMEVTVYH) was kindly provided by Dr M Parkhurst, NCI, $\mathrm{NIH}$. For pulsing, cells were rested overnight in CM with dissolved peptide in final concentrations of 10 , $10^{-1}, 10^{-3}$, and $10^{-5} \mu \mathrm{g} / \mathrm{ml}$ at $37^{\circ} \mathrm{C}$ and $5 \% \mathrm{CO}_{2}$. DC were then washed three times before using them as stimulators. 
Stimulation of CTL clones by DC and melanoma cells and subsequent FACS analysis for the detection of TCR downregulation and intracellular IFN- $\gamma$ expression Before stimulation, $\mathrm{T}$ cells were plated into 48-well plates (costar) at a concentration of $10^{6}$ cells per well. Then, washed DC or melanoma cells were added into each well at a stimulator: effector ratio of 1:3. After $6 \mathrm{~h}$ of coincubation, the cells were transferred to $5 \mathrm{ml}$ tubes (Falcon, Becton Dickinson, Franklin Lakes, NJ, USA) and stained for TCR surface expression and IFN- $\gamma$ intracellular expression. For TCR staining, cells were washed once in FACS buffer (phosphate buffer plus 5\% FCS; Biofluids) and then stained with anti-CD3, -CD8, -CD4, and -TCR mAbs (20 min, on ice, in the dark). For intracellular IFN- $\gamma$ staining, Brefeldin A (Sigma, St Louis, MO, USA) was added to the CTL $1 \mathrm{~h}$ after the stimulation at a final concentration of $10 \mu \mathrm{g} / \mathrm{ml}$. After five additional hours of incubation, the cells were washed once with PBS and incubated for $10 \mathrm{~min}$ in PBS containing $1 \mathrm{mM}$ EDTA. Two additional washes were performed and then the cells were stained in the dark with anti-CD3 and -CD8, -CD4 mAbs for $20 \mathrm{~min}$ on ice. Afterwards, permeabilization solution was added (Becton Dickinson, San Jose, CA, USA) followed by IFN- $\gamma$ staining for $30 \mathrm{~min}$. After staining, cells were washed in $4 \mathrm{ml}$ of ice-cold FACS buffer and analyzed using a FACScalibur flow cytometer and CellQuest software (Becton Dickinson). At least 30000 events were acquired for each sample. The following mAbs were used: anti-TCR alpha/beta-FITC, anti-IFN- $\gamma$-FITC, antiCD3-PerCP, anti-CD8-APC, and anti-CD4-APC (Becton Dickinson).

\section{Statistical analysis}

Lymphocytes, DC, or tumor cell populations were gated according to light scatter. In addition, $\mathrm{T}$ cells were gated according to CD3 and CD8 staining for JR6C12, S6G3, and CK3H6 CTL or CD3 and CD4 staining for F11 CD4+ clone. Expression of CD80, CD83, CD86, HLA-DR, HLA-class I (HLA-A2), and gp100 is presented as the average of the mean fluorescence intensities of individual experiments. Percentages of gp100-positive and IFN- $\gamma$-positive cells represent cells staining above the 97th percentile of the negative control. TCR downregulation was calculated according to the following formula: $f \mathrm{TCR}_{\mathrm{down}}=\left(\mathrm{TCR}_{\text {meanMFI }}\right.$ nonstimulated-TCRmeanMFI stimulated) $/ \mathrm{TCR}_{\text {meanMFI }}$ nonstimulated $\times 100 \%$. TCR $_{\text {meanMFI }}$ nonstimulated represents the mean MFI of TCR expression in JR6C12 without any stimulation and $\mathrm{TCR}_{\text {meanMFI }}$ stimulated represents the mean MFI of TCR expression after the respective stimulation. Paired, two-tailed Student's $t$-tests were performed to compare bead-uptake (mDC versus iDC); cytokine concentrations in supernatants (iDC versus mDC; $\mathrm{DC}-\mathrm{rVV}$ versus $\mathrm{DC}+\mathrm{rVV}$ ), gp100 expression, expression of HLA-DR, CD80, CD83, CD86, HLA-A2, TCR downregulation; and IFN- $\gamma$ expression (both in JR6C12 and F11).

\section{Acknowledgements}

This work was supported by the Dr-Mildred-ScheelStiftung für Krebsforschung, Deutsche Krebshilfe (D.N.).

\section{References}

1 Bhardwaj N, Friedman SM, Cole BC, Nisanian AJ. Dendritic cells are potent antigen-presenting cells for microbial superantigens. J Exp Med 1992; 175: 267-273.

2 Bhardwaj N. Processing and presentation of antigen by dendritic cells: implications for vaccines. Trends Mol Med 2001; 7: 388-394.

3 Albert ML, Sauter B, Bhardwaj N. Dendritic cells acquire antigen from apoptotic cells and induce class I-restricted CTLs. Nature 1998; 392: 86-89.

4 Fontaneau JF, Larsson M, Bhardwaj N. Dendritic cell-dead-cell interactions: implications and relevance for immunotherapy. J Immunother 2001; 24: 294-304.

5 Bronte $\mathrm{V}$ et al. Antigen expression by dendritic cell correlates with the therapeutic effectiveness of a model recombinant poxvirus tumor vaccine. Proc Natl Acad Sci USA 1997; 94(7): 3183-3188.

6 Bhardwaj $\mathrm{N}$ et al. Influenza virus-infected dendritic cells stimulate strong proliferative and cytolytic responses from human CD8+ T cells. J Clin Invest 1994; 94: 797-807.

$7 \mathrm{Kim}$ CJ et al. Dendritic cells infected with poxviruses encoding MART-1/MelanA sensitize T lymphocytes in vitro. J Immunother 1997; 20: 276-286.

$8 \mathrm{Kim} \mathrm{CJ}$ et al. Use of recombinant poxviruses to stimulate antimelanoma T cell reactivity. Ann Surg Oncol 1998; 5: 64-76.

9 Schutz A et al. Immunogenicity of nonreplicating recombinant vaccinia expressing HLA-A201 targeted or complete MART-1/ MelanA antigen. Cancer Gene Ther 2001; 8: 655-661.

10 Sette A et al. The relationship between class I binding affinity and immunogenicity of potential cytotoxic $\mathrm{T}$ cell epitopes. J Immunol 1994; 153: 5586-5592.

11 Kawakami $\mathrm{Y}$ et al. Identification of the immunodominant peptides of the MART-1 human melanoma antigen recognized by the majority of HLA-A2-restricted tumor infiltrating lymphocytes. J Exp Med 1994; 180: 347-352.

12 Kawakami Yet al. Recognition of multiple epitopes in the human melanoma antigen gp100 by tumor-infiltrating $\mathrm{T}$ lymphocytes associated with in vivo tumor regression. J Immunol 1995; 154: 3961-3968.

13 Parkhurst MR et al. Improved induction of melanoma reactive CTL with peptides from the melanoma antigen gp100 modified at HLA-A*0201 binding residues. J Immunol 1996; 157: 2539-2548.

14 Valmori D et al. Diversity of the fine specificity displayed by HLA-A*0201-restricted CTL specific for the immunodominant Melan-A/MART-1 antigenic peptide. J Immunol 1998; 161: 6956-6962.

15 Rivoltini L et al. A superagonist variant of peptide MART-1/ Melan A27-35 elicits anti-melanoma CD8+ T cells with enhanced functional characteristics: implications for more effective immunotherapy. Cancer Res 1999; 59: 301-306.

16 Valmori $\mathrm{D}$ et al. Optimal activation of tumor-reactive $\mathrm{T}$ cells by selected antigenic peptide analogues. Int Immunol 1999; 11: 1971-1980.

17 Salgaller ML, Marincola FM, Cormier JN, Rosenberg SA. Immunization against epitopes in the human melanoma antigen gp100 following patient immunization with synthetic peptides. Cancer Res 1996; 56: 4749-4757.

18 Rosenberg SA et al. Immunologic and therapeutic evaluation of a synthetic tumor associated peptide vaccine for the treatment of patients with metastatic melanoma. Nat Med 1998; 4: 321-327.

19 Iezzi G, Karjalainen K, Lanzavecchia A. The duration of antigenic stimulation determines the fate of naive and effector T-cells. Immunity 1998; 8: 89-95.

20 Lanzavecchia A, Iezzi G, Viola A. From TCR engagement to T-cell activation: a kinetic view of $\mathrm{T}$ cell behavior. Cell 1999; 96: $1-4$. 
21 Larsson $\mathrm{M}$ et al. Requirement of mature dendritic cells for efficient activation of influenza A-specific memory CD8+ T cells. Immunol 2000; 165: 1182-1190.

22 Steinman RM \& Dhodapkar M. Active immunization against cancer with dendritic cells: the near future. Int J Cancer 2001; 94: 459-473.

23 Jonuleit $\mathrm{H}$ et al. A comparison of two types of dendritic cells as adjuvants for the induction of melanoma-specific $\mathrm{T}$ cell responses in humans following intranodal injection. Int J Cancer 2001; 93: 243-251.

24 Engelmayer $\mathrm{J}$ et al. Vaccinia virus inhibits the maturation of human dendritic cells: a novel mechanism of immune evasion. J Immunol 1999; 163: 6762-6768.

25 Smith GL et al. Vaccinia virus immune evasion. Immunol Rev 1997; 159: 137-154.

26 Tsujisaki $M$ et al. Fine specificity and idiotype diversity of the murine anti-HLA- A2, A28 monoclonal antibodies CR11-351 and KS1. Transplantation 1988; 45: 632-639.

27 Bettinotti $M$ et al. Stringent allele/epitope requirements for MART-1/Melan A immunodominance: implications for peptidebased immunotherapy. J Immunol 1998; 161: 877-889.

28 Nielsen MB et al. Status of activation of circulating vaccineelicited CD8+ T cells. J Immunol 2000; 165: 2287-2296.

29 Monsurro' V et al. Functional heterogeneity of vaccine-induced CD8+ T cells. J Immunol 2002; 168: 5933-5942.

30 Dudley ME et al. Adoptive transfer of cloned melanoma-reactive $\mathrm{T}$ lymphocytes for the treatment of patients with metastatic melanoma. J Immunother 2001; 24: 363-373.

31 Dudley ME, Nishimura MI, Holt AKC, Rosenberg SA. Anti-tumor immunization with a minimal peptide epitope (G9-209-2M) leads to a functionally heterogeneous CTL response. J Immunother 1999; 22: 288-298.

32 Lee $\mathrm{KH}$ et al. Increased vaccine-specific $\mathrm{T}$ cell frequency after peptide-based vaccination correlates with increased susceptibility to in vitro stimulation but does not lead to tumor regression. I Immunol 1999; 163: 6292-6300.

33 Ridge JP, Di Rosa F, Matzinger P. A conditioned dendritic cell can be a temporal bridge between a CD4+ T-helper and a T-killer cell [see comments]. Nature 1998; 393: 474-478.

34 Gallucci S, Lolkema M, Matzinger P. Natural adjuvants: endogenous activators of dendritic cells. Nat Med 1999; 5: 1249-1255.

35 Lapointe $\mathrm{R}$ et al. Retrovirally transduced human dendritic cells can generate $\mathrm{T}$ cells recognizing multiple MHC class I and class II epitopes from the melanoma antigen glycoprotein 100. J Immunol 2001; 167: 4758-4764.

36 Parmiani G et al. Cancer immunotherapy with peptide-based vaccines: what have we achieved? Where are we going? J Natl Cancer Inst 2002; 94: 805-818.

37 Kim CJ, Parkinson DR, Marincola FM. Immunodominance across the HLA polymorphism: implications for cancer immunotherapy. J Immunother 1997; 21: 1-16.

38 Marincola FM, Jaffe EM, Hicklin DJ, Ferrone S. Escape of human solid tumors from $\mathrm{T}$ cell recognition: molecular mechanisms and functional significance. Adv Immunol 2000; 74: 181-273.

39 Ferrone S, Marincola FM. Loss of HLA class I antigens by melanoma cells: molecular mechanisms, functional significance and clinical relevance. Immunol Today 1995; 16: 487-494.

40 Rosenberg SA et al. Immunization of patients with metastatic melanoma using recombinant adenoviruses encoding the MART-1 or gp100 melanoma antigens. J Natl Cancer Inst 1998; 90: 1894-1899.

41 Restifo NP, Rosenberg SA. Developing recombinant and synthetic vaccines for the treatment of melanoma. Curr Opin Oncol 1999; 11: 50-57.

42 Coller HA et al. Expression analysis with oligonucleotide microarrays reveals that MYC regulates genes involved in growth, cell cycle, signaling and adhesion. Proc Natl Acad Sci USA 2000; 28: 3260-3265.
43 Wang $\mathrm{R}$ et al. Induction of CD4(+) T cell-dependent CD8(+) type 1 responses in humans by a malaria DNA vaccine. Proc Natl Acad Sci USA 2001; 98: 10817-10822.

44 Janetzki $S$ et al. Immunization of cancer patients with autologous cancer-derived heat shock protein gp96 preparations: a pilot study. Int I Cancer 2000; 88: 232-238.

45 Hsueh EC et al. Active immunotherapy by reinduction with a polyvalent allogeneic cell vaccine correlates with improved survival in recurrent metastatic melanoma. Ann Surg Oncol 2002; 9: 486-492.

46 Shiku $\mathrm{H}$ et al. Development of a cancer vaccine: peptides, proteins and DNA. Cancer Chemother Pharmacol 2000; 46: S77-S82.

47 Kirk CJ, Mule JJ. Gene-modified dendritic cells for use in tumor vaccines. Hum Gene Ther 2000; 11: 797-806.

48 Yang $S$ et al. Dendritic cells infected with a vaccinia vector carrying the human gp100 gene simultaneously present multiple specificities and elicit high-affinity $\mathrm{T}$ cells reactive to multiple epitopes and restricted by HLA-A2 and -A3. J Immunol 2000; 164: 4204-4211.

49 Prabakaran I et al. Mature CD83(+) dendritic cells infected with recombinant gp100 vaccinia virus stimulate potent antimelanoma T cells. Ann Surg Oncol 2002; 9: 411-418.

50 Smith SG et al. Human dendritic cells genetically engineered to express a melanoma polyepitope DNA vaccine induce multiple cytotoxic T cell responses. Clin Cancer Res 2001; 7: 4253-4261.

51 Cella $\mathrm{M}$ et al. Ligation of CD40 on dendritic cells triggers production of high levels of interleukin-12 and enhances T-cell stimulatory capacity: T-T help via APC activation. I Exp Med 1996; 184: 747-752

52 Monsurro' V et al. Kinetics of TCR use in response to repeated epitope-specific immunization. J Immunol 2001; 166: 5817-5825.

53 Granucci F et al. Inducible IL-2 production by dendritic cells revealed by global gene expression analysis. Nat Immunol 2001; 2: 882-888.

54 Andrews DM et al. Infection of dendritic cells by murine cytomegalovirus induces functional paralysis. Nat Immunol 2001; 2: 1077-1084.

55 Sauter B et al. Consequences of cell death: exposure to necrotic tumor cells, but no primary tissue cells or apoptotic cells, induces the maturation of immunostimulatory dendritic cells. J Exp Med 2000; 191: 434.

56 Larsson $\mathrm{M}$ et al. Efficiency of cross presentation of vaccinia virusderived antigens by human dendritic cells. Eur J Immunol 2001; 31: 3432-3442.

57 Sercarz EE et al. Dominance and crypticity of $\mathrm{T}$ cell antigenic determinants. Annu Rev Immunol 1993; 11: 729-766.

58 Niedermann G et al. Contribution of proteasome-mediated proteolysis to the hierarchy of epitopes presented by major histocompatibility complex class I molecules. Immunity 1995; 2: 289-299.

59 Valmori D et al. Induction of potent antitumor CTL responses by recombinant vaccinia encoding a melan-A peptide analogue. J Immunol 2000; 164: 1125-1131.

60 Speiser DE et al. In vivo activation of melanoma-specific CD8(+)T cells by endogenous tumor antigen and peptide vaccines. A comparison to virus-specific T cells. Eur J Immunol 2002; 32: 731-741.

61 Sallusto F, Lanzavecchia A. Efficient presentation of soluble antigen by cultured human dendritic cells is maintained by granulocyte/macrophage colony-stimulating factor plus interleukin 4 and down-regulated by tumor necrosis factor alpha. J Exp Med 1994; 179: 1109-1118.

62 Lapointe $\mathrm{R}$ et al. Human dendritic cells require multiple activation signals for the efficient generation of tumor antigenspecific T lymphocytes. Eur I Immunol 2000; 30: 3291-3298.

63 Cormier JN et al. Heterogeneous expression of melanoma-associated antigens (MAA) and HLA-A2 in metastatic melanoma in vivo. Int J Cancer 1998; 75: 517-524. 